\title{
A Trigonometrical Approach to Morley's Observation
}

\author{
IoAnnis Gasteratos ${ }^{1}$, Spiridon Kuruklis ${ }^{2}$ and Thedore Kuruklis ${ }^{3}$ \\ ${ }^{1}$ Department of Mathematics and Statistics, Boston University, \\ Boston, MA 02215, USA. \\ igaster@bu.edu \\ ${ }^{2}$ Eurobank, Group Information and IT Security, \\ 14234 Athens, Greece.

\begin{abstract}
Simple trigonometrical arguments verify that in a triangle the trisectors, proximal to sides respectively, meet at the vertices of an equilateral triangle by showing that the length of each side is $8 \mathrm{R}$ times the sines of the angles between the sides of the triangle and the trisectors that determine it, where $\mathrm{R}$ is the radius of the circumcircle of the triangle. The 27 meeting points of the trisectors, proximal to a side, determine 18 such equilaterals, which in pairs share a vertex having two collinear sides and the third parallel. Hence these points are located 6 by 6 on three triples of parallel lines.
\end{abstract}

\section{RESUMEN}

Argumentos trigonométricos simples verifican que en un triángulo los trisectores, próximos a los lados respectivamente, se encuentran en los vértices de un triángulo equilátero mostrando que la longitud de cada lado es $8 \mathrm{R}$ veces los senos de los ángulos entre los lados del triángulo y los trisectores que lo determinan, donde $\mathrm{R}$ es el radio del circuncírculo del triángulo. Los 27 puntos de encuentro de los trisectores, próximos a un lado, determinan 18 tales equiláteros, que a pares comparten un vértice teniendo dos lados colineales y el tercero paralelo. Luego estos puntos están ubicados 6 por 6 en tres triples de líneas paralelas.

Keywords and Phrases: Angle trisection, proximal trisector, triangle trisectors, Morley's theorem, Morley triangle, Morley's magic, Morley's miracle, Morley's mystery.

2010 AMS Mathematics Subject Classification: 00A05, 00A08 


\section{Introduction}

One of the infamous contemporary problems in mathematics refers to the angle trisection in a triangle. The problem appeared suddenly around 1900, when Frank Morley made a shocking observation, known since then as Morley's theorem, usually expressed with the statement: In a triangle, the trisectors of its angles, proximal to sides respectively, meet at the vertices of an equilateral triangle.

The above theorem is considered among the most unexpected discoveries in mathematics, as strangely went unnoticed during the ages, even though it expresses a property for trisectors analog to bisectors. Ancient Greeks studied the triangle in depth and they could have discovered it, but simply ignored it. More than one hundred years since its discovery and a very respectable number of publications, several authored by distinguished mathematicians, we are still struggling to fully comprehend it. Words like magic, miracle, mystery, or paradox have appeared in titles of several articles. Notably, Morley's theorem has been included in the list of The hundred greatest theorems.[1]

Morley inferred it while studying the behavior of cardioids from an observation that plainly asserts: In a triangle, the trisectors proximal to a side intersect on three sets of three parallel lines forming equilateral triangles... Thus, if we take the interior trisectors of the angles of a triangle, the points where those proximal to a side meet form an equilateral triangle. [13, p.469]

In Fig.1 appear 27 equilaterals. Their placement reveals a structure with startling symmetry, where an impressive number of overlapping and interconnected equilaterals are arranged with common vertices and parallel or collinear sides. Their existence, in fact with arrangement, is interpreted as evidence of regularity in the behavior of angle trisectors in a triangle, like the incenter and the excenters of a triangle express regularity in the behavior of its angle bisectors.[11]

Visual inspection easily verifies that only 18 from the 27 triangles determined by the meeting points of trisectors of all three angles, proximal to sides respectively, are equilateral and they are called Morley triangles.

Fig.2 illustrates the trisectors of $\angle A B C=3 \beta, 0<\beta<60^{\circ}$. The proximal to side $B C$ trisector is the one (BT) which coincides with side $B A$ after two rotations around $B$ towards $B A$ by $\angle C B T$. Note that the inclinations of the proximal trisectors to the corresponding proximal sides are $\beta, 60^{\circ}-\beta$ and $60^{\circ}+\beta$.

The used formulation of Morley's theorem does not specify the type of trisectors, intersecting in pairs at the vertices of an equilateral. This becomes crucial in the presence of many triangles formed by the intersections of trisectors, proximal to sides respectively. However, several from these triangles - but not all - are equilateral. As the theorem can be valid for other types of trisectors this ambiguity may have been deliberately used, although most publications have focused only on the interior trisectors. In contrast to the elementary, sharp and clean statement of the theorem, approaches dealing with the observation remain abstract or in higher mathematics, where it was 


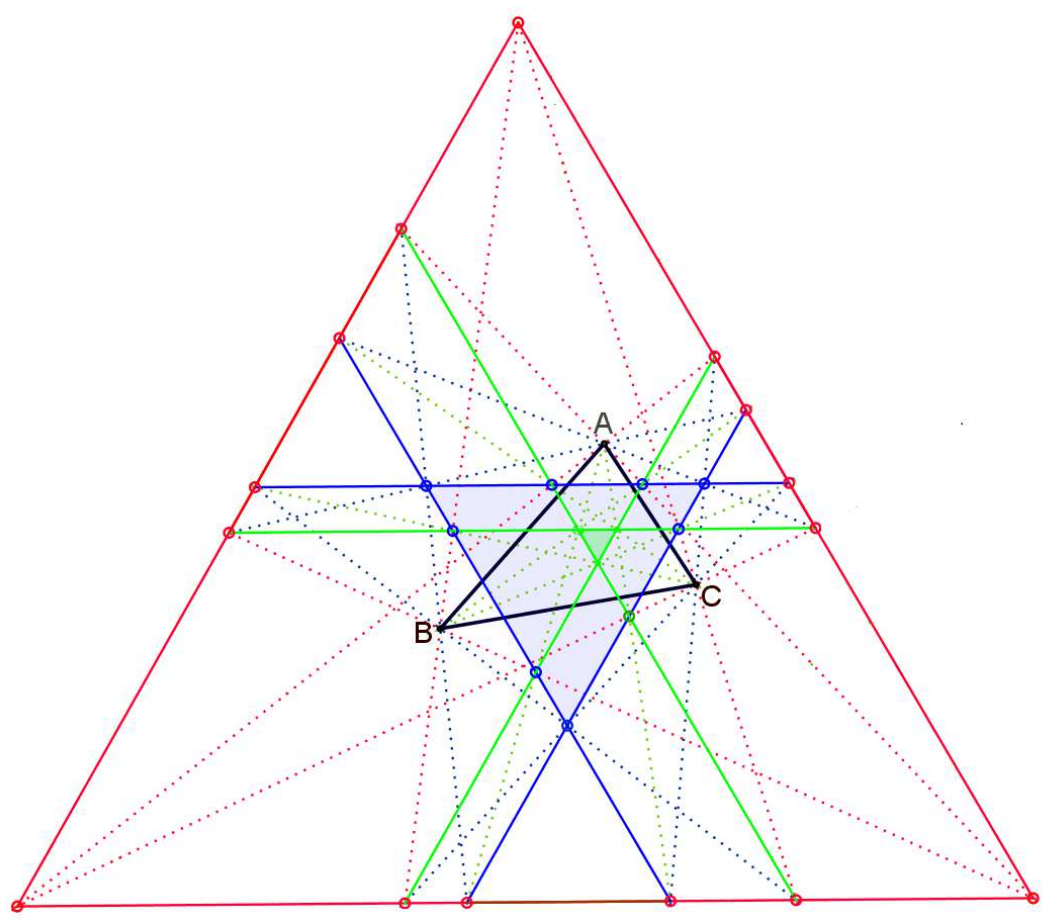

Figure 1: Fig.1: The 27 intersections of trisectors, proximal to sides, determine 18 equilaterals

discovered. [8],[12], [16]

In this presentation, we prove several instances of Morley's theorem yielding the 18 Morley triangles. In fact, it is shown: The side length of a Morley triangle is $8 R$ times the sin of the angles between the sides of the triangle and the trisectors that determine it, where $R$ is the radius of the circumcircle of the triangle. Then, utilizing the arrangement of these triangles, we establish Morley's observation about the alignment of the intersections of trisectors proximal to a side. The approach relies on the following trigonometrical property that combines the sine and cosine laws.

Proposition: In a $\triangle \mathrm{STV}$ with $\angle \mathrm{STV}=\phi$, if $\mathrm{ST}=\mathrm{p} \sin \theta$ and $\mathrm{TV}=\mathrm{p} \sin \omega$, where $\phi+\theta+\omega=180^{\circ}$, then $\mathrm{SV}=\mathrm{p} \sin \phi$ while $\angle \mathrm{SVT}=\theta$ and $\angle \mathrm{TSV}=\omega$.

Proof. In $\Delta$ STV by applying the law of cosines we get (SV $)^{2}=(T S)^{2}+(T V)^{2}-2(T S)(T V) \cos \phi=p^{2} \sin ^{2} \phi$ since $\sin ^{2} \theta+\sin ^{2} \omega-2 \sin \theta \sin \omega \cos \phi=\sin ^{2} \phi$ by the law of cosines in the triangle with sides $\sin \theta$, $\sin \omega$ and $\sin \phi$. Thus $S V=p \sin \phi$.

Now from the law of $\operatorname{sines} \mathrm{ST} / \sin (\angle \mathrm{SVT})=\mathrm{TV} / \sin (\angle \mathrm{TSV})=\mathrm{SV} / \sin \phi . A \mathrm{~S} \mathrm{SV}=\mathrm{p} \sin \phi, \mathrm{ST}=\mathrm{p} \sin \theta$ and $\mathrm{TV}=\mathrm{p} \sin \omega$, we get $\sin \theta=\sin (\angle \mathrm{SVT}), \sin \omega=\sin (\angle \mathrm{TSV})$. Therefore, $\angle \mathrm{SVT}=\theta$ or $\angle \mathrm{SVT}=180^{\circ}-\theta$ and $\angle \mathrm{TSV}=\omega$ or $\angle \mathrm{TSV}=180^{\circ}-\omega$. Since $\phi+\theta+\omega=180^{\circ}$ and $\phi+\angle \mathrm{SVT}+\angle \mathrm{TSV}=180^{\circ}$, only the case $\angle \mathrm{SVT}=\theta$ and $\angle \mathrm{TSV}=\omega$ may hold. 


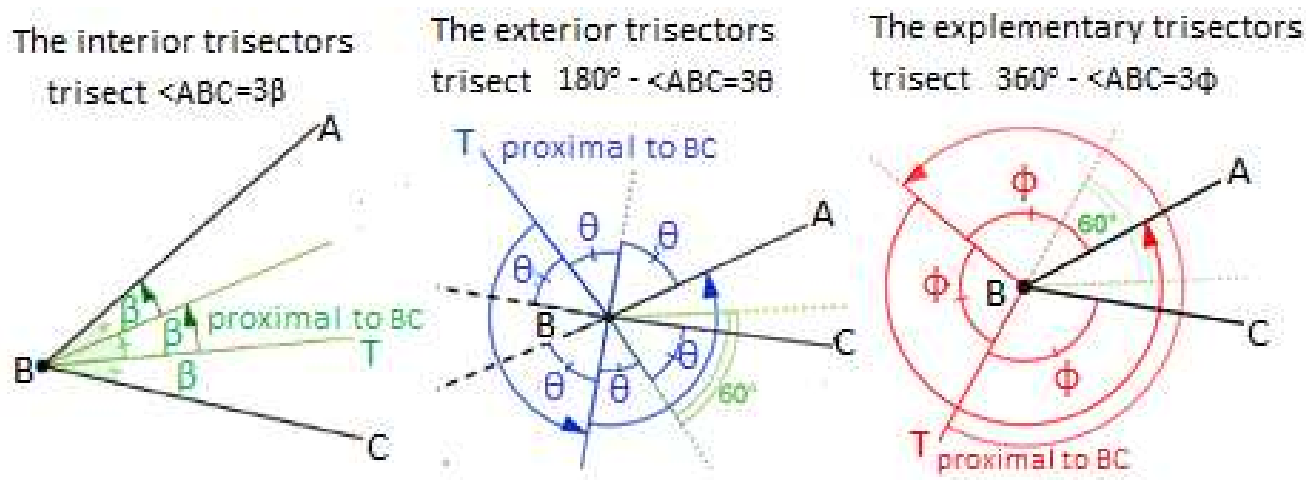

Figure 2: Fig.2: The three types of trisectors

Given a $\triangle A B C$, with $\angle A=3 \alpha, \angle B=3 \beta$ and $\angle C=3 \gamma$, where $\alpha+\beta+\gamma=60^{\circ}$, we establish that $\triangle A^{\prime} B^{\prime} C^{\prime}$ is equilateral, where $A^{\prime}, B^{\prime}$ and $C^{\prime}$ are the intersections of trisectors proximal to sides of $\triangle A B C$ respectively, by showing $A^{\prime} B^{\prime}=B^{\prime} C^{\prime}=C^{\prime} A^{\prime}$. To prove this we apply the above proposition to the adjacent triangles $\Delta A^{\prime} C B^{\prime}, \Delta B^{\prime} A C^{\prime}$ and $\Delta C^{\prime} B A^{\prime}$, formed by a side of $\Delta A^{\prime} B^{\prime} C^{\prime}$ and the trisectors that determine it. The lengths of the trisectors are found from the surrounding triangles $\triangle B A^{\prime} C$, $\triangle C B^{\prime} A$ and $\triangle A C^{\prime} B$ after the sides are expressed by the formulas $A B=2 R \sin 3 \gamma, B C=2 R \sin 3 \alpha$, $A C=2 R \sin 3 \beta$, obtained from the law of sines.

In the appearing expressions, it is convenient to represent angles $\theta+60^{\circ}$ and $60^{\circ}-\theta$ as $\theta^{+}$ and $\theta^{-}$respectively. Hence $\theta^{+}=60^{\circ}+\theta$ and $\theta^{-}=60^{\circ}-\theta$, while $\theta^{++}=120^{\circ}+\theta$ and $\theta^{-+}=120^{\circ}-\theta$. Also, the formula

$$
\sin 3 \theta=4 \sin \left(60^{\circ}-\theta\right) \sin \theta \sin \left(60^{\circ}+\theta\right)=4 \sin \theta^{-} \sin \theta \sin \theta^{+}
$$

will be used to simplify expressions. As $\sin 3 \theta=3 \sin \theta-4 \sin ^{3} \theta$ the above follows after factoring out the right hand side. In the article Are These The Most Beautiful? Hofstadter is quoted that would have given a very high score to Morley's theorem as it follows from this trigonometrical identity. $[15]$

In the sequel, Morley triangles are grouped according to the type of trisectors that determine them and only representatives of groups are examined. The groups are: The primitive triangles formed by the same type of trisectors, the mix triangles formed properly by one type of trisectors of an angle and another type of trisectors of the other two angles, and the complete triangles formed by trisectors of one distinct type for each angle. To avoid degenerate cases in which some of the considered triangles are not formed, in the sequel we assume that the angles of the given triangle are different and not multiples of $30^{\circ}$. 


\section{The 18 Morley triangles}

In this section, we will prove that the 18 Morley triangles are indeed equilaterals by producing formulas for the lengths of their sides.

\section{Primitive Morley triangles (3)}

The primitive triangles are formed exclusively by the intersections of same type trisectors. Hence the two pairs of trisectors determining one of its sides form the same three angles with the corresponding sides of $\triangle A B C$. In the sequel, we find an expression of the length for one side. As this is independent of the side then all sides have the same length and so the specific Morley triangle is equilateral.

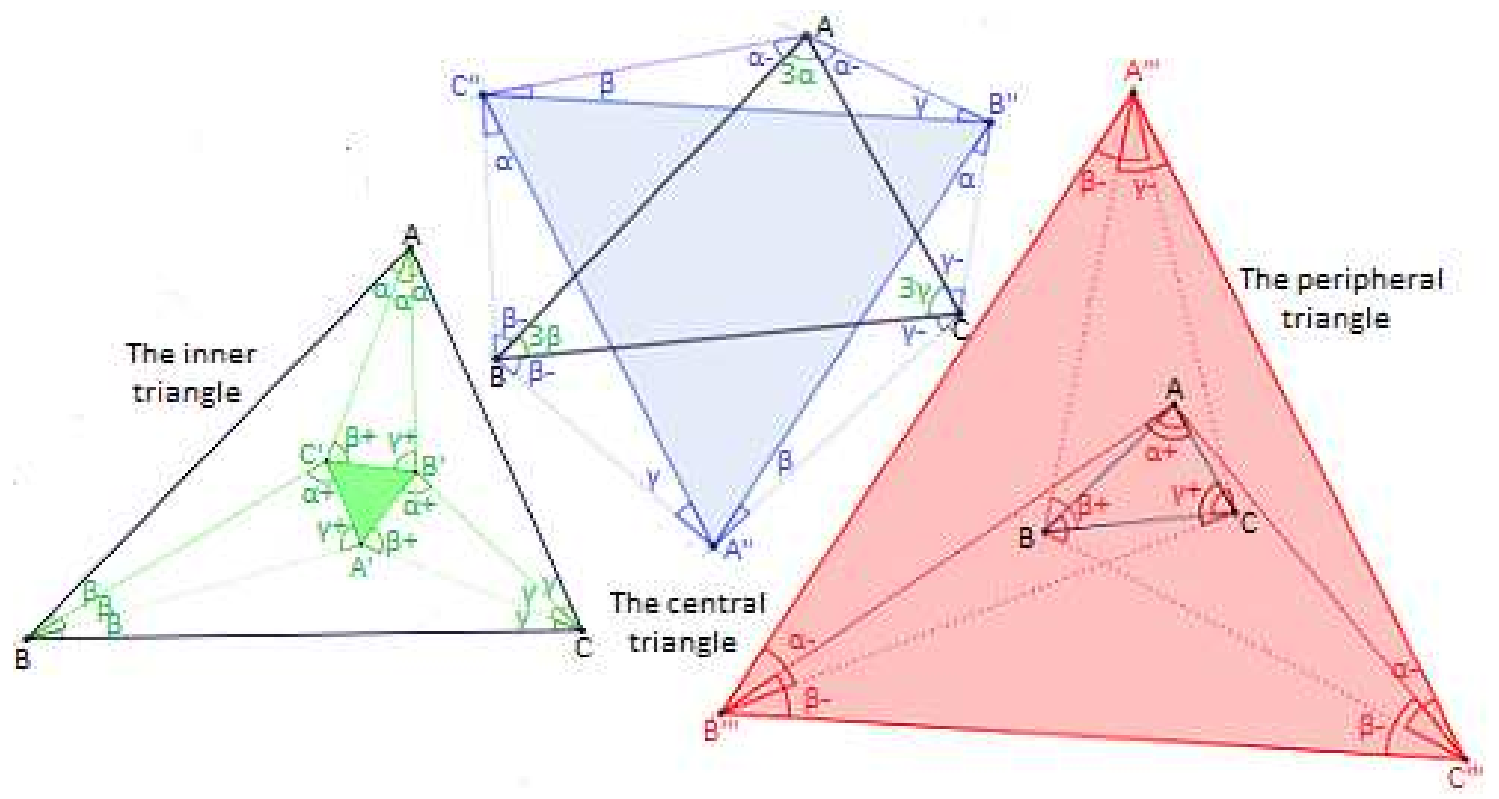

Figure 3: Fig.3: The primitive Morley triangles

Theorem 0.1. In a triangle, the same type trisectors of its angles, proximal to sides respectively, meet at the vertices of a corresponding equilateral.

Proof. Assume that the intersections of the interior, exterior and explementary trisectors, proximal to the sides, $B C, C A$ and $A B$, meet at $A^{\prime}, B^{\prime}$ and $C^{\prime}, A^{\prime \prime}, B^{\prime \prime}$ and $C^{\prime \prime}, A^{\prime \prime \prime}, B^{\prime \prime \prime}$ and $C^{\prime \prime \prime}$, defining $\Delta \mathrm{A}^{\prime} \mathrm{B}^{\prime} \mathrm{C}^{\prime}, \Delta \mathrm{A}^{\prime \prime} \mathrm{B}^{\prime \prime} \mathrm{C}^{\prime \prime}$ and $\Delta \mathrm{A}^{\prime \prime \prime} \mathrm{B}^{\prime \prime \prime} \mathrm{C}^{\prime \prime \prime}$, called inner triangle, central triangle and peripheral triangle respectively.

The proximal trisectors that determine one of their sides form angles with the corresponding 
sides of $\triangle A B C$ equal to $\alpha, \beta$ and $\gamma$, for the inner, or $\alpha^{-}, \beta^{-}$and $\gamma^{-}$, for the central, or $\alpha^{+}, \beta^{+}$and $\gamma^{+}$, for the peripheral triangle, regardless of the side.

a. The lengths of the interior trisectors determining side $B^{\prime} C^{\prime}$ are found from the law of sines in $\triangle A B^{\prime} C$ and $\triangle B C^{\prime} A$ :

$$
A B^{\prime} / \sin \gamma=A C / \sin \left(180^{\circ}-\alpha-\gamma\right)=2 R \sin 3 \beta / \sin \beta^{-}=8 R \sin \beta \sin \beta^{+}
$$

so $A B^{\prime}=8 R \sin \beta \sin \beta^{+} \sin \gamma$. Similarly, $A C^{\prime}=8 R \sin \gamma \sin \gamma^{+} \sin \beta$.

Then in $\triangle B^{\prime} A C^{\prime}, \angle B^{\prime} A C^{\prime}=\alpha, A B^{\prime}=p \sin \beta^{+}, A C^{\prime}=p \sin \gamma^{+}$, where $p=8 R \sin \beta \sin \gamma$. As $\alpha+\beta^{+}+\gamma^{+}=$ $180^{\circ}, B^{\prime} C^{\prime}=p \sin \alpha=8 R \sin \beta \sin \gamma \sin \alpha$ while $\angle A B^{\prime} C^{\prime}=\gamma^{+}$and $\angle A C^{\prime} B^{\prime}=\beta^{+}$. We conclude that $\Delta A^{\prime} B^{\prime} C^{\prime}$ is equilateral.

b. The lengths of the exterior trisectors determining side $\mathrm{B}^{\prime \prime} \mathrm{C}^{\prime \prime}$ are found from the law of sines in $\triangle A B^{\prime \prime} C$ and $\triangle B C^{\prime \prime} A$ :

$$
A B^{\prime \prime} / \sin \gamma^{-}=A C / \sin \left(180^{\circ}-\alpha^{-}-\gamma^{-}\right)=2 R \sin 3 \beta / \sin \beta^{+}=8 R \sin \beta \sin \beta^{-}
$$

and so $A B^{\prime \prime}=8 R \sin \beta \sin \beta^{-} \sin \gamma^{-}$. Similarly, $A C^{\prime \prime}=8 R \sin \gamma \sin \gamma^{-} \sin \beta^{-}$.

Then in $\triangle B^{\prime \prime} A C^{\prime \prime}, \angle B^{\prime \prime} A C^{\prime \prime}=2 \alpha^{-}+3 \alpha=\alpha^{++}, A B^{\prime \prime}=p \sin \beta, A C^{\prime \prime}=p \sin \gamma$, where $p=8 R \sin \beta^{-} \sin \gamma^{-}$. As $\alpha^{++}+\beta+\gamma=180^{\circ}, B^{\prime \prime} C^{\prime \prime}=p \sin \alpha^{++}=8 R \sin \alpha^{-} \sin \beta^{-} \sin \gamma^{-}$while $\angle A B^{\prime \prime} C^{\prime \prime}=\gamma$ and $\angle A C^{\prime \prime} B^{\prime \prime}=\beta$. We conclude that $\Delta \mathrm{A}^{\prime \prime} \mathrm{B}^{\prime \prime} \mathrm{C}^{\prime \prime}$ is equilateral.

c. The lengths of the explementary trisectors determining side $B^{\prime \prime \prime} C^{\prime \prime \prime}$ are found from the law of sines in $\triangle A B^{\prime \prime \prime} C$ and $\triangle B C^{\prime \prime \prime} A$ :

$$
A B^{\prime \prime \prime} / \sin \gamma^{+}=A C / \sin \left(180^{\circ}-\alpha^{+}-\gamma^{+}\right)=2 R \sin 3 \beta / \sin \beta=8 R \sin \beta^{+} \sin \beta^{-}
$$

so $A B^{\prime \prime \prime}=8 R \sin \beta^{+} \sin \beta^{-} \sin \gamma^{+}$. Similarly, $A C^{\prime \prime \prime}=8 R \sin \gamma^{+} \sin \gamma^{-} \sin \beta^{+}$.

Then in $\Delta \mathrm{B}^{\prime \prime \prime} \mathrm{A} \mathrm{C}^{\prime \prime \prime}, \angle \mathrm{B}^{\prime \prime \prime} \mathrm{A} \mathrm{C}^{\prime \prime \prime}=2 \alpha^{+}-3 \alpha=\alpha^{-+}, A \mathrm{~B}^{\prime \prime \prime}=\mathrm{p} \sin \beta^{-}, A C^{\prime \prime \prime}=p \sin \gamma^{-}$, where $\mathrm{p}=$ $8 \mathrm{R} \sin \beta^{+} \sin \gamma^{+}$. As $\alpha^{-+}+\beta^{-}+\gamma^{-}=180^{\circ}$,

$\mathrm{B}^{\prime \prime \prime} \mathrm{C}^{\prime \prime \prime}=\mathrm{p} \sin \alpha^{-+}=8 \mathrm{R} \sin \alpha^{+} \sin \beta^{+} \sin \gamma^{+}$while $\angle A \mathrm{~B}^{\prime \prime \prime} \mathrm{C}^{\prime \prime \prime}=\gamma^{-}$and $\angle A \mathrm{C}^{\prime \prime \prime} \mathrm{B}^{\prime \prime \prime}=\beta^{-}$. We conclude that $\Delta \mathrm{A}^{\prime \prime \prime} \mathrm{B}^{\prime \prime \prime} \mathrm{C}^{\prime \prime \prime}$ is equilateral.

\section{Mix Morley triangles (9)}

The mix triangles are formed by the intersections of same type trisectors of an angle combined with a different type trisectors of the other two. Hence, they share a vertex with a primitive triangle. For a short statement of the next theorem we define the mixable type of explementary, interior and exterior trisectors to be the interior, exterior and explementary trisectors respectively.

Theorem 0.2. In a triangle, the same type trisectors of an angle and the corresponding mixable type trisectors of the other two, proximal to sides respectively, meet at the vertices of an equilateral.

Proof. a. The intersections of the explementary trisectors of an angle and the interior trisectors of the other two proximal to sides respectively, form three triangles, referred as mix inner triangles since each of them shares a vertex with the inner triangle. We will study only one representative from them. Consider the explementary trisectors of $\angle \mathrm{B}$ and the interior trisectors of the other two. 
Assume the interior trisectors proximal to $A C$ meet at $B^{\prime}$ while the explementary and the interior proximal to $\mathrm{BC}$ and $\mathrm{AB}$ meet at $\mathrm{B}_{\mathrm{C}}$ and $\mathrm{B}_{\mathrm{A}}$ defining $\triangle \mathrm{B}^{\prime} \mathrm{B}_{\mathrm{A}} \mathrm{B}_{\mathrm{C}}$, called $B$-inner triangle as it shares vertex $B^{\prime}$ with the inner triangle. The angles between the trisectors that determine it and the corresponding sides are equal to $\beta^{+}$(explementary) and $\alpha, \gamma$ (interior).

Find the lengths of the trisectors defining $\Delta \mathrm{B}^{\prime} \mathrm{B}_{\mathrm{A}} \mathrm{B}_{\mathrm{C}}$ :

In $\triangle B_{B} A$, as $\angle B B_{C} A=180^{\circ}-\alpha-\beta^{+}=\gamma^{+}$,

$$
\mathrm{BB}_{\mathrm{C}} / \sin \alpha=\mathrm{AB}_{\mathrm{C}} / \sin \beta^{+}=\mathrm{AB} / \sin \gamma^{+}=2 \mathrm{R} \sin 3 \gamma / \sin \gamma^{+}=8 \mathrm{R} \sin \gamma \sin \gamma^{-}
$$

and so $\mathrm{BB}_{\mathrm{C}}=8 \mathrm{R} \sin \gamma \sin \gamma^{-} \sin \alpha$ and $\mathrm{AB}_{\mathrm{C}}=8 \mathrm{R} \sin \gamma \sin \gamma^{-} \sin \beta^{+}$.

Similarly, in $\triangle \mathrm{CB}_{\mathrm{A}} \mathrm{B}, \mathrm{BB}_{\mathrm{A}}=8 \mathrm{R} \sin \alpha \sin \alpha^{-} \sin \gamma$ and $\mathrm{CB}_{\mathrm{A}}=8 \mathrm{R} \sin \alpha \sin \alpha^{-} \sin \beta^{+}$.

In $\triangle A B^{\prime} C$, as $\angle A B^{\prime} C=180^{\circ}-\alpha-\gamma=\beta^{++}$,

$$
\mathrm{AB}^{\prime} / \sin \gamma=\mathrm{CB}^{\prime} / \sin \alpha=A C / \sin \beta^{-}=2 \mathrm{R} \sin 3 \beta / \sin \beta^{-}=8 \mathrm{R} \sin \beta \sin \beta^{+}
$$

and $\mathrm{so} A \mathrm{~B}^{\prime}=8 \mathrm{R} \sin \beta \sin \beta^{+} \sin \gamma$ and $\mathrm{CB}^{\prime}=8 \mathrm{R} \sin \beta \sin \beta^{+} \sin \alpha$.

Now in $\triangle B_{C} A B^{\prime}, A B^{\prime}=8 R \sin \beta \sin \beta^{+} \sin \gamma=p \sin \beta$ and

$A B_{C}=8 R \sin \gamma \sin \gamma^{++} \sin \beta^{+}=p \sin \gamma^{++}$where $p=8 R \sin \beta^{+} \sin \gamma$. As $\alpha+\beta+\gamma^{++}=180^{\circ}, B^{\prime} B_{C}=$ $8 R \sin \beta^{+} \sin \gamma \sin \alpha$ while $\angle B_{C} B^{\prime} A=\gamma^{++}$. Similarly, in $\triangle B_{A} C B^{\prime}, B^{\prime} B_{C}=8 R \sin \beta^{+} \sin \gamma \sin \alpha$ while $\angle B_{A} B^{\prime} C=\alpha^{++}$. Also, in $\triangle B^{A} B_{C}, \angle B_{C} B_{B}=2 \beta^{+}-3 \beta=\beta^{-+}, B_{A}=p \sin \alpha^{-}, B_{C}=p \sin \gamma^{-}$, where $p=8 R \sin \alpha \sin \gamma$. As $\beta^{-+}+\alpha^{-}+\gamma^{-}=180^{\circ}, B_{A} B_{C}=p \sin \beta^{-+}=8 R \sin \alpha \sin \gamma \sin \beta^{-+}$while $\angle B_{B} B_{A}=\alpha^{-}, \angle B B_{A} B_{C}=\gamma^{-}$. Since $\sin \beta^{-+}=\sin \beta^{+}$, we conclude that $\Delta B^{\prime} B_{A} B_{C}$ is equilateral.

Corollary 2a. A mix inner and the inner triangle have two collinear sides and the third parallel.

Proof. Consider for instance the B-inner $\triangle \mathrm{B}^{\prime} \mathrm{B}_{\mathrm{A}} \mathrm{B}_{\mathrm{C}}$. It was seen $\angle A \mathrm{~B}^{\prime} \mathrm{B}_{\mathrm{C}}=\gamma^{++}$. As it is equilateral $\angle A B^{\prime} B_{A}=\gamma^{+}$. Also, it was shown in the inner $\triangle A^{\prime} B^{\prime} C^{\prime}$ that $\angle A B^{\prime} C^{\prime}=\gamma^{+}$. Thus, $B^{\prime} B_{A}$ and $B^{\prime} C^{\prime}$ are collinear.

b. The intersections of the interior trisectors of an angle and the exterior trisectors of the other two, proximal to sides respectively, form three triangles, referred as mix central triangles since each of them shares a vertex with the central triangle. We will study only a representative from them. Consider the interior trisectors of $\angle \mathrm{C}$ and the exterior trisectors of the other two. Assume the exterior trisectors proximal to $A B$ meet at $C^{\prime \prime}$ while the interior and the exterior proximal to $B C$ and $A C$ meet at $C_{A}^{\prime \prime}$ and $C_{B}^{\prime \prime}$ defining $\Delta C^{\prime \prime} C_{A}^{\prime \prime} C_{B}^{\prime \prime}$, called $C$-central triangle as it shares vertex $C^{\prime \prime}$ with the central triangle. The angles between the trisectors that determine it and the corresponding sides are equal to $\gamma$ (interior) and $\alpha^{-}, \beta^{-}$(exterior).

Find the lengths of the trisectors defining $\triangle C^{\prime \prime} C_{A}^{\prime \prime} C_{B}^{\prime \prime}$ :

In $\triangle C A C_{B}^{\prime \prime}$, as $\angle C_{B}^{\prime \prime} A C=2 \alpha^{-}+3 \alpha=\alpha^{++}$and so $\angle C C_{B}^{\prime \prime} A=\beta$. Hence

$$
A C_{B}^{\prime \prime} / \sin \gamma=\mathrm{CB}_{\mathrm{A}}^{\prime \prime} / \sin \alpha^{++}=A C / \sin \beta=2 \mathrm{R} \sin 3 \beta / \sin \beta=8 \mathrm{R} \sin \beta^{-} \sin \beta^{+}
$$

and so $A C_{B}^{\prime \prime}=8 R \sin \beta^{-} \sin \beta^{+} \sin \gamma$ and $C C_{B}^{\prime \prime}=8 R \sin \beta^{-} \sin \beta^{+} \sin \alpha^{++}$.

Similarly, in $\triangle \mathrm{CBC}_{\mathrm{A}}^{\prime \prime}, \mathrm{BC}_{\mathrm{A}}^{\prime \prime}=8 \mathrm{R} \sin \alpha^{-} \sin \alpha^{+} \sin \gamma$ and $\mathrm{CC}_{\mathrm{A}}^{\prime \prime}=8 \mathrm{R} \sin \alpha^{-} \sin \alpha^{+} \sin \beta^{++}$. Also in $\triangle \mathrm{AC} \mathrm{C}^{\prime \prime} \mathrm{B}$, 


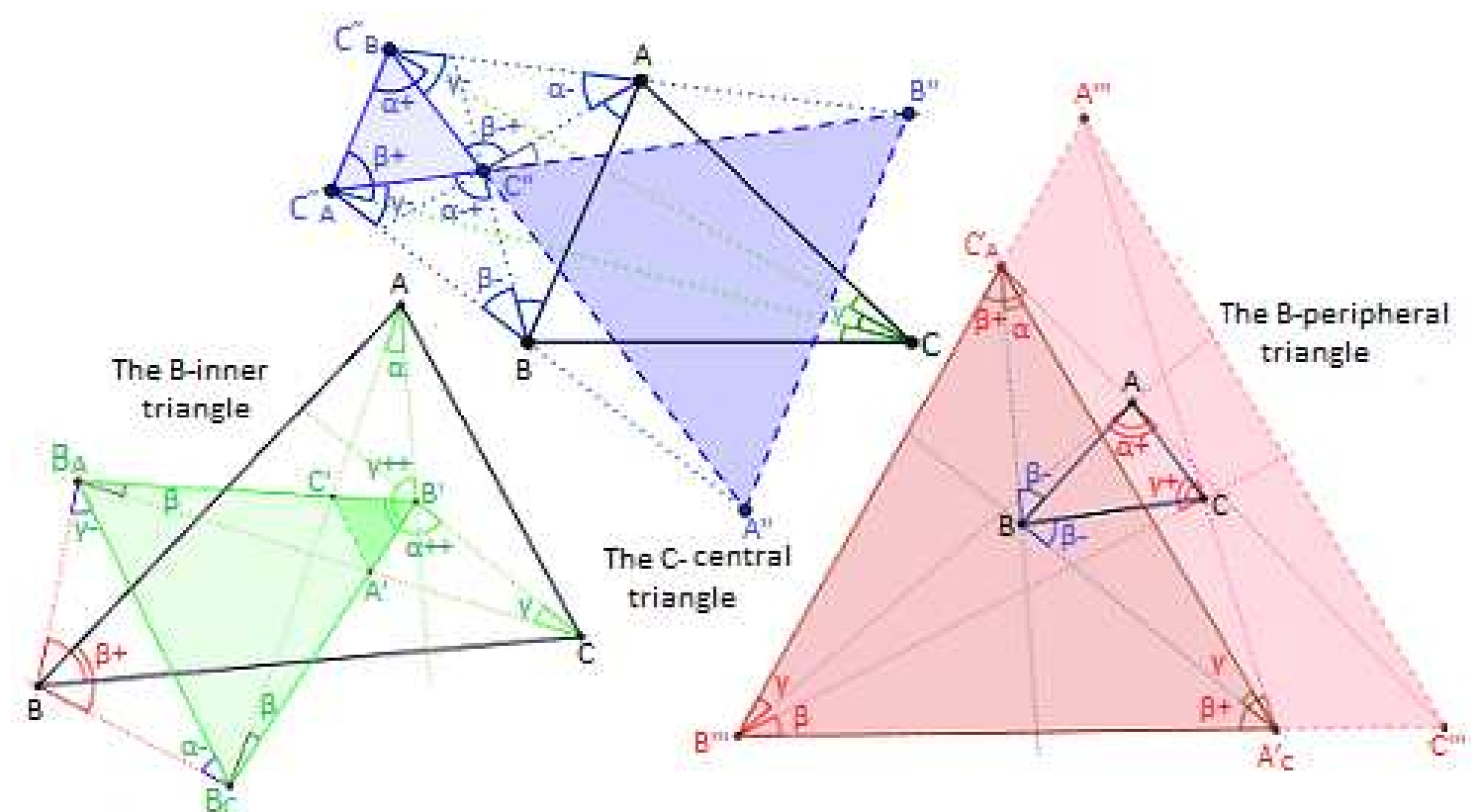

Figure 4: Fig.4: Mix Morley triangles

as $\angle A C^{\prime \prime} B=180^{\circ}-\beta^{-}-\alpha^{-}=\gamma^{-+}$,

$$
\mathrm{AC}^{\prime \prime} / \sin \beta^{-}=\mathrm{BC}^{\prime \prime} / \sin \alpha^{-}=\mathrm{AB} / \sin \gamma^{-+}=8 \mathrm{R} \sin 3 \gamma / \sin \gamma=8 \mathrm{R} \sin \gamma \sin \gamma^{-}
$$

and so $A C^{\prime \prime}=8 \mathrm{R} \sin \gamma \sin \gamma^{-} \sin \beta^{-}$and $\mathrm{BC}^{\prime \prime}=8 \mathrm{R} \sin \gamma \sin \gamma^{-} \sin \alpha^{-}$.

Then in $\triangle C_{B}^{\prime \prime} A C^{\prime \prime}, \angle C_{B}^{\prime \prime} A C^{\prime \prime}=\alpha^{-}, A C_{B}^{\prime \prime}=p \sin \beta^{-+}, A C^{\prime \prime}=p \sin \gamma^{-}$where $p=8 R \sin \gamma \sin \beta^{-}$. As $\alpha^{-}+\beta^{-+}+\gamma^{-}=180^{\circ}, C_{B}^{\prime \prime} C^{\prime \prime}=p \sin \alpha^{-}=8 R \sin \gamma \sin \beta^{-} \sin \alpha^{-}, \angle A C_{B}^{\prime \prime} C^{\prime \prime}=\gamma^{-}, \angle A C^{\prime \prime} C_{B}^{\prime \prime}=\beta^{-+}$. Similarly in $\triangle \mathrm{C}_{A}^{\prime \prime} B \mathrm{C}^{\prime \prime}, \mathrm{C}_{A}^{\prime \prime} \mathrm{C}^{\prime \prime}=8 \mathrm{R} \sin \gamma \sin \beta^{-} \sin \alpha^{-}$and $\angle B \mathrm{~B}_{A}^{\prime \prime} \mathrm{C}^{\prime \prime}=\gamma^{-}, \angle B \mathrm{C}^{\prime \prime} \mathrm{C}_{A}^{\prime \prime}=\alpha^{-+}$. Also in $\triangle \mathrm{C}_{A}^{\prime \prime} \mathrm{C}_{\mathrm{B}}^{\prime \prime}$, $\angle C_{A}^{\prime \prime} C_{B}^{\prime \prime}=\gamma, C_{B}^{\prime \prime}=p \sin \beta^{+}, C_{A}^{\prime \prime}=p \sin \alpha^{+}$, where $p=8 R \sin \alpha^{-} \sin \beta^{-}$. As $\gamma+\beta^{+}+\alpha^{+}=180^{\circ}$, $\mathrm{C}_{A}^{\prime \prime} \mathrm{C}_{\mathrm{B}}^{\prime \prime}=\mathrm{p} \sin \gamma=8 \mathrm{R} \sin \alpha^{-} \sin \beta^{-} \sin \gamma$ and $\angle \mathrm{CC}_{\mathrm{A}}^{\prime \prime} \mathrm{C}_{\mathrm{B}}^{\prime \prime}=\beta^{+}, \angle \mathrm{CC}_{\mathrm{B}}^{\prime \prime} \mathrm{C}_{\mathrm{A}}^{\prime \prime}=\alpha^{+}$. We conclude that $\triangle \mathrm{C} \mathrm{C}_{\mathrm{A}}^{\prime \prime} \mathrm{C}_{\mathrm{B}}^{\prime \prime}$ is equilateral.

Corollary 2b. A mix central and the central triangle have two collinear sides and the third parallel.

Proof. Consider for instance the mix central $\triangle C^{\prime \prime} C_{A}^{\prime \prime} C_{B}^{\prime \prime}$. It was seen $\angle A C^{\prime \prime} C_{B}^{\prime \prime}=\beta^{-+}$. Also, it was shown that in the central $\triangle A^{\prime \prime} B^{\prime \prime} C^{\prime \prime}, \angle A C^{\prime \prime} B^{\prime \prime}=\beta$. As it is equilateral, $\angle A C^{\prime \prime} C_{B}^{\prime \prime}+\angle A C^{\prime \prime} B^{\prime \prime}+$ $\angle B^{\prime \prime} C^{\prime \prime} A^{\prime \prime}=180^{\circ}$. So $C_{B}^{\prime \prime} C^{\prime \prime}$ and $C^{\prime \prime} A^{\prime \prime}$ are collinear.

c. The intersections of the exterior trisectors of an angle and the explementary trisectors of the other two, proximal to sides respectively, define three triangles, referred as mix peripherals since each of them shares a vertex with the peripheral triangle. We will study only one representative from them. Consider the exterior trisectors of $\angle \mathrm{B}$ and the explementary trisectors of $\angle \mathrm{C}$ and $\angle \mathrm{A}$. 
Assume that the explementary trisectors proximal to $A C$ meet at $B^{\prime \prime \prime}$ while the explementary and the exterior proximal to $A B$ and $B C$ meet at $C_{A}^{\prime}$ and $A_{C}^{\prime}$ defining $\Delta B^{\prime \prime \prime} C_{A}^{\prime} A_{C}^{\prime}$, called B-peripheral triangle as it shares vertex $\mathrm{B}^{\prime \prime \prime}$ with the peripheral. The angles between the trisectors that determine it and the corresponding sides are equal to $\beta^{-}$(exterior) and $\alpha^{+}, \gamma^{+}$(explementary).

The lengths of trisectors that determine $\Delta \mathrm{B}^{\prime \prime \prime} \mathrm{C}_{A}^{\prime} A_{C}^{\prime}$ are:

In $\triangle B C_{A}^{\prime} A, \angle A B C_{A}^{\prime}=\beta^{-}, \angle B A C^{\prime}=180^{\circ}-\alpha^{+}=\alpha^{-+}$and so $\angle B C_{A}^{\prime} A=\gamma^{-}$,

$$
A C_{A}^{\prime} / \sin \beta^{-}=B C_{A}^{\prime} / \sin \alpha^{-+}=A B / \sin \gamma^{-}=2 R \sin 3 \gamma / \sin \gamma^{-}=8 R \sin \gamma \sin \gamma^{+} ;
$$

thus $A C_{A}^{\prime}=8 R \sin \gamma \sin \gamma^{+} \sin \beta^{-}$and $B C_{A}^{\prime}=8 R \sin \gamma \sin \gamma^{+} \sin \alpha^{-+}$.

Similarly, in $\triangle C A_{C}^{\prime} B, C A_{C}^{\prime}=8 R \sin \alpha \sin \alpha^{+} \sin \beta^{-}$and $B A_{C}^{\prime}=8 R \sin \alpha \sin \alpha^{+} \sin \gamma^{-+}$.

Also in $\triangle A B^{\prime \prime \prime} C$, as $\angle A B^{\prime \prime \prime} C=180^{\circ}-\alpha^{+}-\gamma^{+}=\beta$,

$$
A B^{\prime \prime \prime} / \sin \gamma^{+}=\mathrm{CB}^{\prime \prime \prime} / \sin \alpha^{+}=A C / \sin \beta=2 R \sin 3 \beta / \sin \beta=8 R \sin \beta^{+} \sin \beta^{-} ;
$$

thus $A B^{\prime \prime \prime}=8 R \sin \beta^{+} \sin \beta^{-} \sin \gamma^{+}$and $C^{\prime \prime \prime}=8 R \sin \beta^{+} \sin \beta^{-} \sin \alpha^{+}$.

Then in $\triangle C_{A}^{\prime} A B^{\prime \prime \prime}, \angle C_{A}^{\prime} A B^{\prime \prime \prime}=180^{\circ}-2 \alpha^{+}+3 \alpha=\alpha^{+}, A C_{A}^{\prime}=p \sin \gamma$, $A B^{\prime \prime \prime}=p \sin \beta^{+}$where $p=8 R \sin \beta^{-} \sin \gamma^{+}$. As $\alpha^{+}+\gamma+\beta^{+}=180^{\circ}$, $B^{\prime \prime \prime} C_{A}^{\prime}=p \sin \alpha^{+}=8 R \sin \beta^{-} \sin \gamma^{+} \sin \alpha^{+}, \angle A B^{\prime \prime \prime} C_{A}^{\prime}=\gamma, \angle A C_{A}^{\prime} B^{\prime \prime \prime}=\beta^{+}$. Similarly, in $\triangle C B^{\prime \prime \prime} A_{C}^{\prime}$, $B^{\prime \prime \prime} A_{C}^{\prime}=8 R \sin \beta^{-} \sin \alpha^{+} \sin \gamma^{+}, \angle C B^{\prime \prime \prime} A_{C}^{\prime}=\alpha, \angle C A_{C}^{\prime} B^{\prime \prime \prime}=\beta^{+}$.

Also, in $\triangle A_{C}^{\prime} B C_{A}^{\prime}$, as $\angle A_{C}^{\prime} B C_{A}^{\prime}=2 \beta^{-}+3 \beta=\beta^{++}, B C_{A}^{\prime}=p \sin \gamma, B A_{C}^{\prime}=p \sin \alpha$, where $p=$ $8 \mathrm{R} \sin \gamma^{+} \sin \alpha^{+}$. As $\beta^{++}+\alpha+\gamma=180^{\circ}$,

$C_{A}^{\prime} A_{C}^{\prime}=p \sin \beta^{++}=8 R \sin \gamma^{+} \sin \alpha^{+} \sin \beta^{++}$while $\angle B A_{C}^{\prime} C_{A}^{\prime}=\gamma, \angle B C_{A}^{\prime} A_{C}^{\prime}=\alpha$. Since $\sin \beta^{++}=\sin \beta^{-}$, we conclude that $\Delta \mathrm{B}^{\prime \prime \prime} C_{A}^{\prime} A_{C}^{\prime}$ is equilateral.

Corollary 2c. A mix peripheral and the peripheral triangle have two collinear sides and the third parallel.

Proof. Consider for instance the mix peripheral $\triangle B^{\prime \prime \prime} C_{A}^{\prime} A_{C}^{\prime}$. It was seen $\angle A B^{\prime \prime \prime} C_{A}^{\prime}=\gamma$. Also in the peripheral equilateral, it was shown $\angle A B^{\prime \prime \prime} C^{\prime \prime \prime}=\gamma^{-}$. Hence $\angle A B^{\prime \prime \prime} A^{\prime \prime \prime}=\gamma$. Thus, $B^{\prime \prime \prime} C^{\prime} A$ and $\mathrm{B}^{\prime \prime \prime} \mathrm{C}^{\prime \prime \prime}$ are collinear.

\section{Complete Morley triangles (6)}

The complete Morley triangles are formed by the intersections of the interior, exterior and explementary trisectors from each angle, proximal to sides respectively. Apparently, there are $3 \times 2$ such triangles. For example, the interior trisectors of $\angle \mathrm{C}$ combined with the explementary trisectors proximal to $\mathrm{CB}$ or $\mathrm{CA}$ form two different complete triangles.

Theorem 0.3. In a triangle, the trisectors of a distinct type from each angle, proximal to sides respectively, meet at the vertices of an equilateral.

Proof. From the 6 triangles formed by the intersections of the interior, exterior and explementary trisectors from each angle, proximal to sides, we will study only a representative as the rest are 


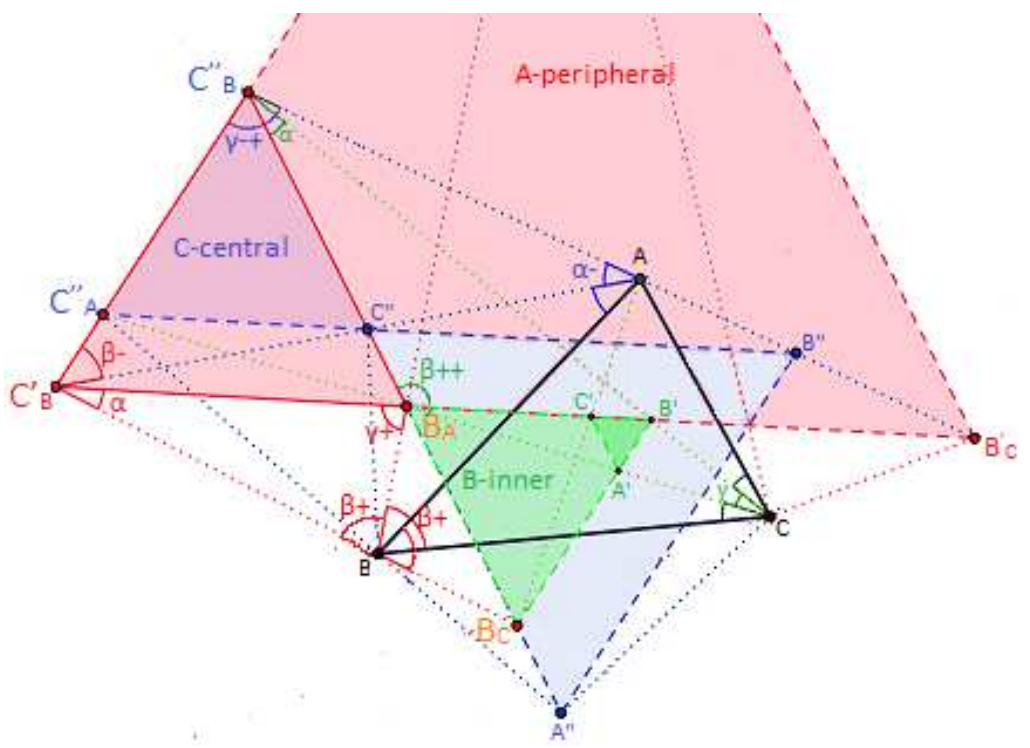

Figure 5: Fig.5: The $\mathrm{B}_{\mathrm{A}}$-complete Morley triangle

similar. Consider for instance the interior trisectors of $\angle \mathrm{C}$, the exterior trisectors of $\angle \mathrm{A}$ and the explementary trisectors of $\angle \mathrm{B}$, proximal to sides respectively. Assume that the interior and the explementary proximal to $C B$ meet at $B_{A}$, the interior with the exterior proximal to $C A$ meet at $C_{B}^{\prime \prime}$ and the exterior with the explementary meet at $C_{B}^{\prime}$ defining $\Delta B_{A} C_{B}^{\prime \prime} C_{B}^{\prime}$ called $B_{A}$-complete triangle as it shares vertex $B_{A}$ with the $B$-inner triangle. It also shares vertex $C_{B}^{\prime \prime}$ with the $C$-central, and vertex $C_{B}^{\prime}$ with the $A$-peripheral triangle. The angles between the trisectors that determine it and the corresponding sides are equal to $\alpha^{-}$(exterior), $\beta^{+}$(explementary) and $\gamma$ (interior).

The lengths of the trisectors that determine it are:

In $\triangle A C_{B}^{\prime} B, \angle B A C_{B}^{\prime}=\alpha^{-}, \angle C_{B}^{\prime} B A=180^{\circ}-\beta^{+}=\beta^{-+}$and so $\angle A C_{B}^{\prime} B=\gamma^{-}$. Hence

$$
\mathrm{AC}_{\mathrm{B}}^{\prime} / \sin \beta^{-+}=\mathrm{C}_{\mathrm{B}}^{\prime} / \sin \alpha^{-}=\mathrm{AB} / \sin \gamma^{-}=2 \mathrm{R} \sin 3 \gamma / \sin \gamma^{-}=8 \mathrm{R} \sin \gamma \sin \gamma^{+}
$$

thus $\mathrm{AC}_{\mathrm{B}}^{\prime}=8 \mathrm{R} \sin \gamma \sin \gamma^{+} \sin \beta^{-+}$and $\mathrm{BC}_{\mathrm{B}}^{\prime}=8 \mathrm{R} \sin \gamma \sin \gamma^{+} \sin \alpha^{-}$.

Similarly, in $\triangle \mathrm{BB}_{\mathrm{A}} \mathrm{C}$, as $\angle \mathrm{B}_{\mathrm{A}} \mathrm{BC}=\beta^{+}, \angle \mathrm{BB}_{\mathrm{A}} \mathrm{C}=\alpha^{+}$,

$$
\mathrm{CB}_{\mathrm{A}} / \sin \beta^{+}=\mathrm{BB}_{\mathrm{A}} / \sin \gamma=\mathrm{BC} / \sin \alpha^{+}=2 \mathrm{R} \sin 3 \alpha / \sin \alpha^{+}=8 \mathrm{R} \sin \alpha \sin \alpha^{-} ;
$$

thus $\mathrm{CB}_{\mathrm{A}}=8 \mathrm{R} \sin \alpha \sin \alpha^{-} \sin \beta^{+}$and $\mathrm{BB}_{\mathrm{A}}=8 \mathrm{R} \sin \alpha \sin \alpha^{-} \sin \gamma$.

Also in $\triangle C C_{B}^{\prime \prime} A$, as $\angle A C C_{B}^{\prime \prime}=\gamma$ and $\angle C_{B}^{\prime \prime} A C=2 \alpha^{-}+3 \alpha=\alpha^{++}, \angle A C_{B}^{\prime \prime} C=\beta$,

$$
\mathrm{CC}_{\mathrm{B}}^{\prime \prime} / \sin \alpha^{++}=A C_{\mathrm{B}}^{\prime \prime} / \sin \gamma=A C / \sin \beta=2 \mathrm{R} \sin 3 \beta / \sin \beta=8 \mathrm{R} \sin \beta^{-} \sin \beta^{+} ;
$$

thus $\mathrm{CC}_{\mathrm{B}}^{\prime \prime}=8 \mathrm{R} \sin \beta^{-} \sin \beta^{+} \sin \alpha^{++}$and $A \mathrm{C}_{\mathrm{B}}^{\prime \prime}=8 \mathrm{R} \sin \beta^{-} \sin \beta^{+} \sin \gamma$.

Then, in $\triangle C_{B}^{\prime \prime} A C_{B}^{\prime}, \angle C_{B}^{\prime \prime} A C_{B}^{\prime}=\alpha^{-}, A C_{B}^{\prime \prime}=p \sin \beta^{-}, A C_{B}^{\prime}=p \sin \gamma^{+}=p \sin \gamma^{-+}$where $p=$ $8 \mathrm{R} \sin \gamma \sin \beta^{-+}$. As $\alpha^{-}+\beta^{-}+\gamma^{-+}=180^{\circ}$,

$\mathrm{C}_{\mathrm{B}}^{\prime \prime} \mathrm{C}_{\mathrm{B}}^{\prime}=\mathrm{p} \sin \alpha^{-}=8 \mathrm{R} \sin \gamma \sin \beta^{-+} \sin \alpha^{-}$while $\angle A \mathrm{C}_{\mathrm{B}}^{\prime} \mathrm{C}_{\mathrm{B}}^{\prime \prime}=\beta^{-}, \angle A C_{\mathrm{B}}^{\prime \prime} \mathrm{C}_{\mathrm{B}}^{\prime}=\gamma^{-+}$. 
Also in $\triangle C_{B}^{\prime} B B_{A}, \angle C_{B}^{\prime} B B_{A}=180^{\circ}-2 \beta^{+}+3 \beta=\beta^{+}, B C_{B}^{\prime}=p \sin \gamma^{+}, B_{A}=p \sin \alpha$ where $p=$ $8 \mathrm{R} \sin \gamma \sin \alpha^{-}$. As $\beta^{+}+\gamma^{+}+\alpha=180^{\circ}$,

$C_{B}^{\prime} B_{A}=p \sin \beta^{+}=8 R \sin \gamma \sin \alpha^{-} \sin \beta^{+}$while $\angle B B_{A} C_{B}^{\prime}=\gamma^{+}$and $\angle B C_{B}^{\prime} B_{A}=\alpha$.

Furthermore, in $\triangle \mathrm{B}_{\mathrm{A}} \mathrm{CC}_{\mathrm{B}}^{\prime \prime}, \angle \mathrm{B}_{\mathrm{A}} \mathrm{CC}_{\mathrm{B}}^{\prime \prime}=\gamma, \mathrm{CC}_{\mathrm{B}}^{\prime \prime}=p \sin \beta^{-}=p \sin \beta^{++}, \mathrm{CB}_{\mathrm{A}}=p \sin \alpha$, where $p=$ $8 \mathrm{R} \sin \alpha^{++} \sin \beta^{+}$. As $\gamma+\beta^{++}+\alpha=180^{\circ}$,

$C_{B}^{\prime \prime} A_{C}=p \sin \gamma=8 R \sin \alpha^{++} \sin \beta^{+} \sin \gamma$ while $\angle C_{A} C C_{B}^{\prime \prime}=120^{\circ}+\beta=\beta^{++}$and

$\angle C C_{B}^{\prime \prime} B_{A}=\alpha$. We conclude that $\triangle B_{A} C_{B}^{\prime \prime} C_{B}^{\prime}$ is equilateral.

Corollary 3. A complete and a mix inner, or a mix central or a mix peripheral triangle, sharing a vertex, have two collinear sides and the third parallel.

Proof. Consider for instance the complete $\triangle B_{A} C_{B}^{\prime \prime} C_{B}^{\prime}$. This shares a vertex with the mix inner $\Delta B^{\prime} B_{A} B_{C}$, the mix central $\Delta C^{\prime \prime} C_{A}^{\prime \prime} C_{B}^{\prime \prime}$, and the mix peripheral $\Delta A^{\prime \prime \prime} B_{C}^{\prime} C_{B}^{\prime}$.

It was shown $\angle B B_{A} C_{B}^{\prime}=\gamma^{+}$. However, in the B-inner $\triangle B^{\prime} B_{A} B_{C}, \angle B B_{A} B_{C}=\gamma^{-}$. Since it is equilateral, $\angle B_{C} B_{A} B^{\prime}=60^{\circ}$. Then $\angle C_{B}^{\prime} B_{A} B^{\prime}=\angle C_{B}^{\prime} B_{A} B+\angle B B_{A} B_{C}+\angle B_{C} B_{A} B^{\prime}=\gamma^{+}+\gamma^{-}+60^{\circ}=180^{\circ}$ and so $C_{B}^{\prime} B_{A}$ and $B_{A} B^{\prime}$ are collinear. Hence, $\Delta B_{A} C_{B}^{\prime \prime} C_{B}^{\prime}$ and $\Delta B^{\prime} B_{A} B_{C}$ have two collinear sides with the third ones parallel.

It was shown $\angle A C_{B}^{\prime \prime} C_{B}^{\prime}=\gamma^{-+}$. As it is equilateral $\angle A C_{B}^{\prime \prime} B_{A}=\gamma^{-}$. However, in the C-central, $\triangle C^{\prime \prime} C_{A}^{\prime \prime} C_{B}^{\prime \prime} \angle A C_{B}^{\prime \prime} C^{\prime \prime}=\gamma^{-}$. Thus, $C_{B}^{\prime \prime} C^{\prime \prime}$ and $C_{B}^{\prime \prime} B_{A}$ are collinear. Hence, $\triangle B_{A} C_{B}^{\prime \prime} C_{B}^{\prime}$ and $\triangle C^{\prime \prime} C_{A}^{\prime \prime} C_{B}^{\prime \prime}$ have two collinear sides with the third parallel.

It was shown $\angle A C_{B}^{\prime} C_{B}^{\prime \prime}=\beta^{-}$. Since is $\triangle B_{A} C_{B}^{\prime \prime} C_{B}^{\prime}$ equilateral, $\angle A C_{B}^{\prime} B_{A}=\beta$. However, in the $B$-peripheral, $\angle B C_{A}^{\prime} A_{C}^{\prime}=\alpha$. Symmetrically, in the $A$-peripheral $\triangle A^{\prime \prime \prime} B_{C}^{\prime} C_{B}^{\prime}, \angle A C_{B}^{\prime} B_{C}^{\prime}=\beta$. Hence $\angle A C_{B}^{\prime} B_{A}=\angle A C_{B}^{\prime} B_{C}^{\prime}$ and so $C_{B}^{\prime} B_{A}$ and $C_{B}^{\prime} B_{C}^{\prime}$ are collinear. Thus $\triangle B_{A} C_{B}^{\prime \prime} C_{B}^{\prime}$ and $\triangle A^{\prime \prime \prime} B_{C}^{\prime} C_{B}^{\prime}$ have two collinear sides with the third parallel.

From the above we infer that a precise formulation of the general Morley's theorem yielding 18 equilaterals is: In a triangle, the same type trisectors of the three angles, the same type trisectors of an angle with its mixable type trisectors of the other two, and the trisectors of a distinct type from each angle, proximal to sides respectively, meet at the vertices of an equilateral.

\section{Arrangement of Morley triangles and alignment of inter- sections of proximal trisectors}

The trisectors of a triangle, proximal to one of its sides, meet at 27 points. Each of them is a common vertex of two Morley triangles which are arranged with two sides collinear and the third parallel.

From corollary 3 , sides $C_{B}^{\prime \prime} B_{A}$ and $C_{B}^{\prime \prime} C_{B}^{\prime}$ of the complete $\Delta B_{A} C_{B}^{\prime \prime} C_{B}^{\prime}$ and the mix central $\Delta C^{\prime \prime} C_{B}^{\prime \prime} C_{A}^{\prime \prime}$ are collinear. Hence, $C_{B}^{\prime \prime}$ and $B_{A}$ lie on the line determined by the side $C^{\prime \prime} A^{\prime \prime}$ of the 
central triangle $\Delta A^{\prime \prime} B^{\prime \prime} C^{\prime \prime}$. Thus, on the line determined by a side of the central triangle lie 6 intersections of trisectors proximal to a side, two of the interior with the exterior trisectors, two of the interior with the explementary trisectors and two between the exterior trisectors of the central triangle.

Also, sides $B_{A} C_{B}^{\prime}$ and $B_{A} B$ of the complete $\triangle B_{A} C_{B}^{\prime \prime} C_{B}^{\prime}$ and the mix inner are collinear. Hence, $\mathrm{C}_{\mathrm{B}}^{\prime \prime}$ and $\mathrm{B}_{\mathrm{A}}$ lie on the line which is determined by a side of the inner triangle. Thus, on the line determined by a side of the inner triangle lie 6 intersections of trisectors proximal to a side, two of the exterior with the explementary trisectors, two of interior with the explementary trisectors and two intersections between interior trisectors of the inner triangle.

Finally, sides $C_{B}^{\prime} C_{B}^{\prime \prime}$ and $C_{B}^{\prime} A^{\prime \prime \prime}$ of the complete $\triangle B_{A} C_{B}^{\prime \prime} C_{B}^{\prime}$ and the mix peripheral triangle $\triangle A^{\prime \prime \prime} B_{C}^{\prime} C_{B}^{\prime}$ are collinear. As the $A$-peripheral and the peripheral have sides $A^{\prime \prime \prime} C_{B}^{\prime}$ and $A^{\prime \prime \prime} B^{\prime \prime \prime}$ collinear, $C_{B}^{\prime \prime}$ and $C_{B}^{\prime}$ lie on side $A^{\prime \prime \prime} B^{\prime \prime \prime}$. Symmetrically $C_{B}^{\prime \prime}$ and $C_{B}^{\prime}$ lie on side $A^{\prime \prime \prime} B^{\prime \prime \prime}$ as well. Thus, on a side of the peripheral triangle lie 6 intersections of trisectors proximal to a side, two of the interior with the exterior trisectors, two of the explementary with the exterior trisectors and two between the explementary trisectors of the peripheral triangle.

Conclude that the intersections of trisectors proximal to a side lie 6 by 6 on three triples of parallel lines intersecting with $60^{\circ}$ angles.

\section{Open Problems}

Next there are three basic questions stemming out from this work inviting further exploration.

1. How many equilaterals do the intersections of trisectors in a triangle determine?

2. Are there lines or circles, beyond Morley's 3 triples of parallel lines, on which the intersections of trisectors lie?

3. Do theorems exist, analog to Morley's theorem regarding angle trisectors, for the side or perpendicular trisectors?

Very special thanks to Gerry Ladas and Thanasis Fokas for their unceasing encouragement to our long efforts in demystifying Morley's mystery.

\section{References}

[1] Paul and Jack Abad, The Hundred Greatest Theorems, in Actes de la confrence JFLA2008.

[2] B. Bollobás, The Art of Mathematics, Cambridge University Press, (2006), 126-127.

[3] Alain Connes, A new proof of Morley's theorem, Publications Mathmatiques de l' IHÉS, (1998), 43-46. 
[4] John Conway, The power of mathematics, In Power, Cambridge University Press, (2006), 36-50.

[5] John Conway, On Morley's Trisector Theorem, The Mathematical Intelligencer, 36, no. 3(2014), 3-3

[6] Edsger W. Dijkstra, A Collection of Beautiful Proofs, Selected Writings on Computing: A personal Perspective, Springer, (1982), 174-183.

[7] Edsger W. Dijkstra, On the design of a simple proof for Morley's Theorem, Programming and Mathematical Method, Volume 88 of the NATO ASI Series, Springer (1992) 3-9.

[8] W. J. Dobbs, Morley's triangle, The Mathematical Gazette, 1938 JSTOR.

[9] Richard K. Guy, The Lighthouse Theorem A Budget of Paradoxes, Amer. Math. Monthly, 114 (2007), 97-141

[10] Clark Kimberling, Central points and central lines in the plane of a triangle, Mathematics Magazine, 67 (1994), 163-187.

[11] Spiridon A. Kuruklis, Trisectors like Bisectors with Equilaterals instead of Points, CUBO 16, 2 (2014), 71-110.

[12] Henri Lebesgue, Sur les n-sectrices d'un triangle [En memoire de Frank Morley (1860-1930)], Enseingnement Math., 38 (1940), 39-58.

[13] Frank Morley, Extensions of Clifford's Chain-Theorem, American J. of Math., 51 (1929), 465472.

[14] Roger Penrose, Morley's trisector theorem, Eureka The Archimedeans Journal, Cambridge, 16 (1953), 6-7.

[15] David Wells, Are These The Most Beautiful? The Mathematical Intelligencer, 12 (1990), $37-41$.

[16] Glanville F. Taylor and L.W. Marr, The six trisectors of each of the angles of a triangle, Proceedings of the Edinburgh Mathematical Society 33 (1913-14), 119-131.

[17] Alexander Bogomolny, Morley's Miracle, Interactive Mathematics Miscellany and Puzzles, http://www.cut-the-knot.org/triangle/Morley/ 